\title{
Suicide and self-harm prevention for people in immigration detention
}

\section{Suicide is the leading cause of premature death for people in the Australian immigration detention network}

$\mathrm{n}$ May this year, the Commonwealth and Immigration Ombudsman released a long-awaited report into suicide and self-harm across the Australian immigration detention network. ${ }^{1}$ All available evidence points to the conclusion that suicide is the leading cause of premature death for detainees across the network. Between 1 July 2010 and 31 December 2012, there were 11 deaths in immigration detention. Five are confirmed suicides. ${ }^{2,3}$

The Ombudsman's inquiry was unable to accurately estimate the incidence of self-harm. While government officials could produce some data on self-harm for specific purposes, such as responding to parliamentary inquiries, it appears that they do not regularly monitor the incidence and prevalence of self-harm among detainees in detention facilities. Notwithstanding, the Ombudsman did find a strong relationship between rises in the average time in detention and self-harm — which has been known for over a decade. ${ }^{4}$ Many detainees have a past history of torture and/or trauma, are concerned for the wellbeing of family and dependents left behind, feel socially isolated and lonely, and feel confined or trapped in the closed detention environment. Lack of resolution of visa status and the prospect of indefinite detention contribute to despair and feelings of hopelessness. Mental distress and despair are clinical correlates of being held in detention. ${ }^{5}$

\section{A specific suicide prevention strategy}

With such complex psychological processes at play, it is all too easy to provide a mental health response that is largely built around the behaviour of detainees. The Ombudsman is right to recommend that coherent mental health policies be integrated consistently across the immigration detention network, proactively addressing risk factors and reinforcing protective factors. ${ }^{1}$ The Ombudsman has also called for a review of the length of time spent in detention and the specific needs of people facing prolonged detention because of policy or security issues. The risk of suicide and self-harm needs to be addressed in specific high-risk groups, such as torture and trauma survivors and unaccompanied minors. The Ombudsman also recommends a specific suicide prevention strategy for people in immigration detention. ${ }^{1}$ Such a strategy should: - be targeted, integrated and coordinated

- identify the best place to position and deploy resources for prevention of self-harm and suicide

- take into account the closed and restricted nature of detention facilities and the explanatory model of detainee behaviour

- include universal, selective and indicated interventions.

1School of Nursing and Midwifery, University of South Australia, Adelaide, SA.

2 Australian Institute for Suicide Research and Prevention,

Griffith University,

Brisbane, QLD.

3 Centre for Developmental Psychiatry and Psychology, Monash University, Melbourne, VIC.

nicholas.procter@ unisa.edu.au

doi: 10.5694/mjal3.10804
Editorial $\mathbf{p} 721$ Research p 776

Universal interventions are pre-emptive and aimed at the overall detainee population; they are tailored to promote protective factors and positive mental health, and to provide information and education on mental healthrelated topics that detainees perceive as relevant. Selective interventions are aimed at detainees who are at greater risk of suicide than the general detainee population and may include specific strategies to assess and offer support to such detainees. Indicated interventions are used for detainees who appear to be at very high or imminent risk of suicide. ${ }^{6}$ The distress and accompanying behaviour must be understood in the context of detention, but it seems quite predictable that interventions would have a limited impact on those facing prolonged, indefinite detention. Knowledge of these and other issues, such as cultural background, is important and should promote:

- an understanding of the factors that heighten self-harm and suicide risk

- identification of factors that protect people from selfharm and suicide risk

- access to external community mental health services that provide culturally and linguistically appropriate care, and coordination and continuity of care. ${ }^{7}$

Due to the indefinite and uncertain nature of immigration detention, all detainees, particularly those facing prolonged detention, are likely to require indicated interventions to prevent suicide. Indicated mental health interventions, while important, probably have a more limited role for detainees subject to prolonged detention. In such cases, these detainees must be transferred to appropriate facilities for mental health treatment.

\section{Articulated and coordinated approaches}

Cultural explanatory models - relating to how detainees understand the concepts of mental health and mental illness; how they perceive themselves and those around them; how they present symptoms; when, how and why they seek help; and what they perceive as a good outcome ${ }^{8}$ - must be considered as the starting point of designing a suicide prevention strategy for immigration detainees. Incorporation of explanatory models helps realise culturally appropriate mental health interventions that incorporate assessment, treatment, case management, and all-of-centre interactions between detainees and service providers. ${ }^{7}$ Explanatory models, such as expression of thoughts, feelings and symptoms, sometimes expressed by metaphor and embodied in personal history and ethnic background, also feature in the culture and social processes of closed detention. Being in despair, in deep distress and frightened

may mean some detainees struggle for words, and when

they fail to find them may express themselves in ways 
government officials and service providers are not used to. The way in which service providers and officials interpret and understand the detainees' dilemma is important. Care must be taken to avoid labelling distress as "bad behaviour" or "putting on a show" and invalidating distress and distressing experiences. ${ }^{9}$ It is essential that such information be included in the education and training of staff working with detainees. Closed environments can become "total institutions" and have adverse effects on psychological and emotional functioning. This affects the way detainees perceive themselves, others and their immediate surroundings.

Collectively, such considerations should be taken into account for prevention of both suicide and mental ill health. This would form "preventive and protective action" across the immigration detention network for detainees who are at risk. Isolated actions have a much lesser probability of yielding significant population health outcome results than more articulated and coordinated approaches that bring people and services together in a meaningful way. ${ }^{10}$ There are two intersecting requirements for the latter: collection of meaningful data on self-harm and suicidal ideation; and rigorous, independent monitoring of mental health standards and practices for detainees.

\section{Collecting data on self-harm and suicidal ideation.}

Meaningful data on self-harm and suicidal ideation should be characterised by a qualitative dimension that can provide insight on the views of detainees and service providers. This would help to deepen the understanding of a possible explanatory model. We believe that there must be a focus on strategically incorporating assessment of the physical consequences of suicidal behaviour and ascertainment of the possibility of intention to die in suicidal acts. ${ }^{6}$ Intention to die or stop living should be quantified as any degree that is greater than zero. This recognises the ambivalence in suicidal behaviour and the concurrent presence, in the same individual, of other intentions to suicidal acts. In making this point, we acknowledge that intention to die will be subject to deliberate denial or exaggeration. In many cases, it is difficult for the individuals themselves to disentangle the motivational components of a suicidal act. ${ }^{11}$ In addition, interpretation or recall of these motivations can change; over time, individuals often provide very different reconstructions of their self-harming acts compared with their initial reconstructions. ${ }^{11}$ Despite these complexities, deepening and developing our understanding of self-harm and suicidal ideation can help clinicians engage detainees for ongoing therapy and support as an integrated clinically informed response.

Monitoring mental health standards and practices. Working closely with the Immigration Health Advisory Group, bodies such as the Australian Council on Healthcare Standards should be asked to provide independent, objective evidence to assess whether current practices for detainees meet applicable predetermined and published standards. This is not simply a matter of quality assurance or tick-a-box clinical auditing: it is about independent scrutiny of relevant clinical indicators and the quality of mental health care provided to detainees. The recently introduced National Safety and Quality Health Service

Standards - formulated by the Australian Commission on Safety and Quality in Health Care to drive the implementation and use of safety and quality systems and improve the quality of health service provision in Australia $^{12}$ — should apply to detainees.

\section{The longer-term view}

Australian

states and

territories are

working on

strategies to

prevent suicide

in the general

population. We

need to do the

same for people

in held detention

rar

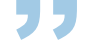

Management of asylum seekers in Australia has gained national and international attention. The intricacies of the current situation are characterised by aspects that go beyond the mere political dimension, and are entrenched with issues that are difficult to prioritise hierarchically, such as ethical values, moral implications, human rights and national interests. Nonetheless, reform is urgently needed. If people continue to be detained under the current arrangements, there is significant scope to investigate in more detail what an episode of self-harm or case of attempted suicide constitutes in the context of held detention. This will go a long way towards improving our understanding of the deeper meaning structures surrounding risk, protective factors associated with suicidal behaviour and ways to favourably strengthen protective factors.

Australian states and territories are working on strategies to prevent suicide in the general population. We need to do the same for people in held detention. Currently, most asylum seekers are facing offshore processing in remote locations such as Nauru, where health and mental health facilities are limited. Prolonged detention in poor conditions directly contributes to mental deterioration. What we do for asylum seekers while they are in detention has the potential to prevent future loss of life. Optimal mental health promotion and suicide prevention strategies can help to prevent self-harm among detainees and help detainees reclaim their lives on release.

Competing interests: Nicholas Procter has received grant funding and sitting fees from the Department of Health and the Department of Immigration and Citizenship. He is a member of the Immigration Detention Health Advisory Group (Mental Health Subgroup) and former member of the Minister's Council on Asylum Seekers and Detention. Diego De Leo has received grant funding and sitting fees from the Department of Health. He is a member of the Australian Suicide Prevention Advisory Council. Louise Newman has received sitting fees from the Department of Immigration and Citizenship and is a member of the Immigration Health Advisory Group. Nicholas Procter, Diego De Leo and Louise Newman are all former members of the Steering Committee for the Commonwealth Ombudsman's Own Motion Investigation into Suicide and Self-harm.

Provenance: Not commissioned; externally peer reviewed.

1 Commonwealth and Immigration Ombudsman. Suicide and self-harm in the immigration detention network. Canberra: Commonwealth Ombudsman, 2013. http://www.ombudsman.gov.au/files/suicide_and_self-harm_ in_the_immigration_detention_network.pdf (accessed Jun 2013).

2 Queensland Courts, Office of the State Coroner. Findings of inquest. File No. 2011/942. 27 May 2013. http:// www.courts.qld.gov.au/_data/assets/pdf_file/0008/184247/cif-hussain-m-20130527.pdf (accessed Aug 2013). 3 Newman LK, Procter NG, Dudley MJ. Suicide and self-harm in immigration detention [editorial]. Med J Aust 2011; 195: 310-311.

4 Robjant K, Hassam R, Katona C. Mental health implications of detaining asylum seekers: systematic review. $\mathrm{Br}$ J Psychiatry 2009; 194: 306-312.

5 Newman L, Procter NG, Dudley M. Seeking asylum in Australia: immigration detention, human rights and mental health care. Australas Psychiatry 2013; 21: 315-320.

6 De Leo D, Burgis S, Bertolote JM, et al. Definitions of suicidal behavior: lessons learned from the WHO/EURO multicentre study. Crisis 2006; 27: 4-15.

7 Department of Health and Ageing. Fourth national mental health plan: an agenda for collaborative government action in mental health 2009-2014. Canberra: Commonwealth of Australia, 2009. http://www.health.gov.au/ internet/publications/publishing.nsf/Content/mental-pubs-f-plan09-toc (accessed Jun 2013).

8 Kleinman A. Patients and healers in the context of culture. Berkeley: University of California Press, 1980.

9 Freeman H, Stansfeld S, editors. The impact of the environment on psychiatric disorder. London: Routledge, 2008.

10 Bertolote JM. Suicide prevention: at what level does it work? World Psychiatry 2004; 3: 147-151.

11 De Leo D. DSM-V and the future of suicidology. Crisis 2011; 32: 233-239.

12 Australian Council on Healthcare Standards. ACHS National Safety and Quality Heath Service Standards. 2012. http://www.achs.org.au/achs-nsqhs-standards (accessed Jun 2013). 